\title{
A hybrid intelligent system for PID controller using in a steel rolling process
}

\author{
José Luis Calvo-Rolle, José Luis Casteleiro-Roca, Héctor Quintián*, María del Carmen Meizoso-Lopez
}

Departamento de Ingeniería Industrial, University of A Coruña, A Coruña, Spain

\section{A R T I C L E I N F O}

\section{Keywords:}

Steel rolling process

Knowledge engineering

PID

Open-loop tuning

Ruled-based system

Hybrid intelligent system

\begin{abstract}
A B S T R A C T
With the aim to improve the steel rolling process performance, this research presents a novel hybrid system for selecting the best parameters for tuning in open loop a PID controller. The novel hybrid system combines rule based system and Artificial Neural Networks. With the rule based system, it is modeled the existing knowledge of the PID controller tuning in open loop and, with Artificial Neural Network, it is completed the rule based model that allow to choose the optimal parameters for the controller. This hybrid model is tested with a long dataset to obtain the best fitness. Finally, the novel research is validated on a real steeling roll process applying the hybrid model to tune a PID controller which set the input speed in each of the gearboxes of the process.
\end{abstract}

(c) 2013 Elsevier Ltd. All rights reserved.

\section{Introduction}

It is well known that there are a lot of industrial process far away from the optimal point of operation (Marlin, 2000). This fact is due to a lot of reasons, but only some of them are very significant in order to get a better performance (Marlin, 2000). Astrom and Hagglud in Åström and Hägglund (2009) indicate that one of the most important fact is to choose the adequate control technique. In this sense, several works have been developed with the aim to improve the behavior and consequently make the process more optimal. For instance (Ko et al., 2011) proposes an efficient control method to minimize process error and to reduce process variance in semiconductor manufacturing, in Chen et al. (2008) is described an intelligent adaptive control system for multiple-input multipleoutput (MIMO) uncertain nonlinear systems and, in Etik et al. (2009) is showed a controlled fuzzy expert system to provide the conditions necessary for operating rooms. Depending of the control technique used different results are achieved. These techniques must be selected based on the desired response of the system. Some of these typical control methods are: PID control is the most common solution for the practical control loops (Åström and Hägglund, 2009), non-linear control (Haddad and Chellaboina, 2011), it would be used when the process is non linear; adaptive control (Sastry and Bodson, 2011), it would be used for process that are non-linear or changes are introduced on it during the operation; Model Predictive Control, it would be used to address process with difficult dynamics (Camacho and Bordons, 2004).

\footnotetext{
* Corresponding author.

E-mail addresses: jlcalvo@udc.es (J.L. Calvo-Rolle), jose.luis.casteleiro@udc.es (J.L. Casteleiro-Roca), hector.quintian@udc.es (H. Quintián), mmeizoso@udc.es (M. del Carmen Meizoso-Lopez).
}

Despite PID controller is applicable in most control loops cases, but for certainly processes it has some limitations. For these processes it is possible to make changes in the controller topology achieving good results. For instance in Astrom and Wittenmark (1994) an adaptive PID controller is implemented for non linear systems or changes in it are made; (Rugh, 1991) introduces the Gain Scheduling concept for non linear systems with predicted variations in the process; in Bahill (1983) is showed the predictive PID controllers to address non-minimum phase systems.

The experience of human experts is used to create rule based systems models (Hayes-Roth et al., 1983; Cimino et al., 2012). Expert people extract rules from a system operation and then they structure it according to the system performance (Hayes-Roth et al., 1983). These methods allow the developed model to emulate the experts behavior in a certain field (Hayes-Roth et al., 1983; Hayes-Roth, 1985), and have been one of the most used methods in both research and operation (Hayes-Roth, 1985). There are several examples of those models, for instance: (Olugu and Wong, 2012) shows an expert fuzzy rule-based system applied to the automotive industry; (Chang et al., 2011) makes a comparison between a rule-based expert system and optimization models in a small drinking water network; in Liu et al. (2010) a rule-based control system design for smart grids were developed. It is possible to create models based on Intelligent Systems. In particular models based on Artificial Neural Networks (ANN) are usually used to improve the fitting of some models (Bishop, 2006). As examples of works where ANN are used to create models are: (Garliauskas, 2004) describes the model created for mapping the evaluation of transmitted information in the biological area; in Stanikunas and Vaitkevicius (2000) is showed the model developed for color constancy based on Four-layer neural network; (Alvarez-Huerta et al., 
2011) shows the model developed to predict the drywell temperature of a nuclear power plant.

Many are the methods and applications that combine rulebased systems and models based on (ANN). For instance (FerreiroGarcia, 2012) shows a model to improve the heat exchanger supervision using neural networks and rule based techniques. In Srivastava et al. (1999) a knowledge-based conceptual neural network is developed for fast voltage contingency selection and ranking. Chaoui et al. (2004) proposes a control strategy based on Artificial Neural Networks for a positioning system with a flexible transmission element, with a rule-based supervisor for online adaptation of the parameters of the reference model.

Usually, the control loops of steel rolling process are conventional, and the operators therefore require no ongoing training and updating. Thus, it is necessary that the improvements should aim to complement the monitoring and control applications with which the operators are fully familiar. There are several previous works which tries to get this improvements (Chen et al., 2010; Wan et al., 2008; Maheral et al., 1995; Sbarbaro-Hofer et al., 1993). In this work a novel hybrid intelligent system for PID controller tuning in open loop is proposed. It is based on a rule based expert system combined with Artificial Neural Networks. With the proposal it is possible to tuning the PID controller with the optimal parameters according with the operation point of the steel rolling process on load.

One approach, to solve the above problems, is to create a generic decision method, based on a conceptual model describing the necessary steps to be achieved in order to obtain the PID controller parameters for open-loop empirical adjustment method. The novel model presented in this study was developed based on six different sets of expressions with highly satisfactory results commonly used in control systems.

The rest of the paper is organized as follows: first, the different intelligent classification method used in this study are describe in Section 2. Section 3 describes the procedure to tune a PID controller in open loop and the different expressions considered for selecting the PID parameters. Section 4 describes the novel hybrid system; its components, dataset, experiments and results are presented. Section 5 presents the empirical application of the novel hybrid system on steel rolling process. Finally the conclusions and future work are present.

\section{Classification methods}

In this section is described briefly the classification techniques are used in the model approach. Three methods were taken into account.

\subsection{Artificial Neural Networks, ANN}

The ANNs (Artificial Neural Networks) are computational algorithms based on the functioning of the human brain. Once of the most used ANN is the MLP (Multilayer Perceptron) (Bishop, 2006). The MLP is composed by one input layer, one or more hidden layers and one output layer (see Fig. 1), all of them made of neurons and pondered connections between neurons of each layer. Applying the Theorem of Universal Approximation (Hornik et al., 1989), can be demonstrated that only one hidden layer is needed to model a nonlinear projection between input and output layer.

A MLP with one hidden layer, can be written mathematically as show in the Eq. (1).

$y_{k}^{p}=F_{k}\left(\sum_{i=1}^{L} w_{i k} F_{i}\left(\sum_{j=1}^{N} w_{j i} x_{j}^{p}+b_{i}\right)+b_{k}\right)$

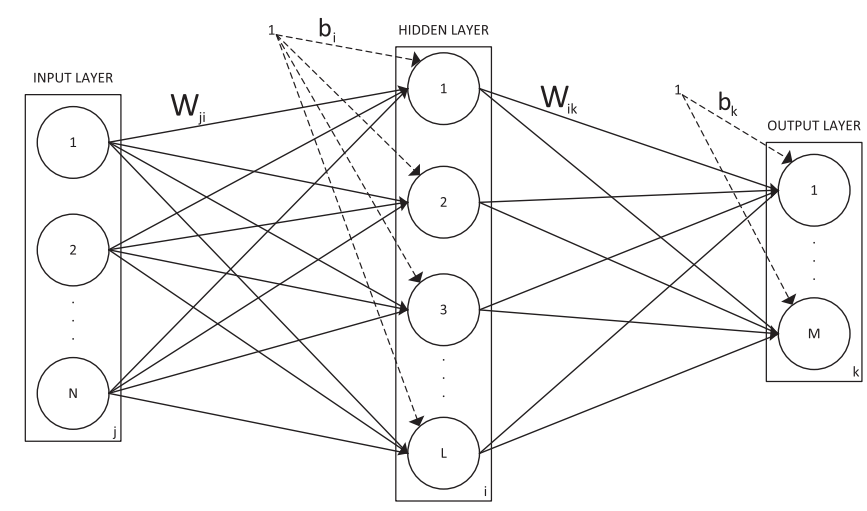

Fig. 1. Architecture of Multilayer Perceptron with 1 hidden layer.

Where:

$F_{k} \rightarrow$ Activation function of neurons of the output layer.

$w_{i k} \rightarrow$ Weight vector of connections from neurons of hidden layer to neurons of output layer.

$b_{k} \rightarrow$ Bias of neurons of the output layer.

$k \rightarrow$ Number of neurons of the output layer.

$F_{i} \rightarrow$ Activation function of neurons of the hidden layer.

$w_{j i} \rightarrow$ Weight vector of connections from neurons of input layer to neurons of hidden layer.

$b_{i} \rightarrow$ Bias of neurons of the hidden layer.

$i \rightarrow$ Number of neurons of the hidden layer.

$x_{j}^{p} \rightarrow$ p-th input pattern.

$j \rightarrow$ Number of neurons of the input layer (equals to dimension of the input data).

$y_{k}^{p} \rightarrow$ Predicted output for the p-th input pattern.

\subsection{Support Vector Machines, SVM}

SVM is described as a statistical learning method based on a structural risk minimization procedure (Cristianini and Scholkopf, 2002). The basic concept of the algorithm is a mapping of the input space into a higher dimensional feature space. Mapping can be done either linearly or non-linearly, according to the used kernel function. In the new feature space, the SVM constructs separating hyperplanes that are optimal in the sense that the classes are separated with the largest margin and minimum classification error. The optimal hyperplane can be written as a combination of a few

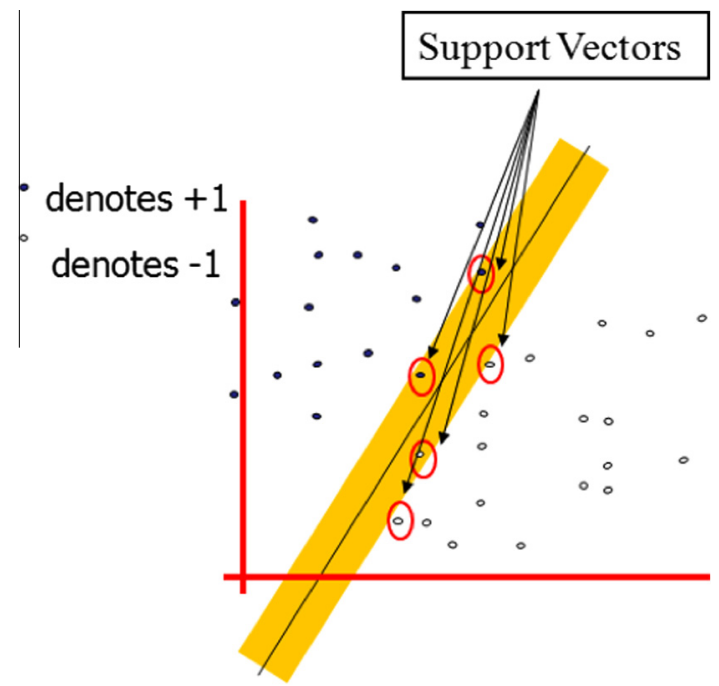

Fig. 2. SVM classification with $\epsilon$-insensitive loss function. 
feature points, which are called the support vectors of the optimal hyper plane (Fig. 2).

Classification of the test sample $x$ is performed by the Eq. (2), where $N$ is the number of training samples, $y_{i}$ is the class label, $\alpha_{i}$ is the Lagrangian multiplier, the elements $x_{i}$ for which $\alpha_{i}>0$ are the support vectors, and $K\left(s_{i}, x\right)$ is the function kernel.

$y=\operatorname{sgn}\left(\sum_{i=1}^{N} \alpha_{i} y_{i} K\left(s_{i}, x\right)\right)$

\subsubsection{Least Squares SVM (LS-SVM)}

Least Square formulation of SVM, are called LS-SVM, in the approximation the solution is obtained by solving a system of linear equations, and it is comparable to SVM in terms of generalization performance Ye and Xiong (2008). In LS-SVM, the $\epsilon$ - insensitive loss function is replaced by a classical squared loss function, which constructs the Lagrangian by solving the linear KarushKuhn-Tucker (KKT) system (Eq. (3)).

$\left[\begin{array}{cc}0 & I_{n}^{T} \\ I_{n} & K+\gamma_{-1} I\end{array}\right]\left[\begin{array}{c}b_{0} \\ b\end{array}\right]=\left[\begin{array}{l}0 \\ y\end{array}\right]$

Where $I_{n}$ is a [ $n \times 1$ ] vector of ones, $T$ means transpose of a matrix or vector, $\gamma$ a weight vector, $b$ regression vector and $b_{0}$ is the model offset.

In LS-SVM, only 2 parameters $(\gamma, \sigma)$ are needed. Where $\sigma$ is the width of the used kernel (Rud and Mining, 2000).

\subsection{Fisher's Linear Discriminant Analysis, FLDA}

This method with Fishers Discriminant Criterion (Belhumeur et al., 1997) is better than other techniques for Discriminant Analysis (Swets and Weng, 1996). It is important to highlight that with this method good results are obtained in many cases, where linear classification is possible. The FLDA carry out discrimination of classes by hyperplanes which are derived from the training data. In the event that there are two classes, the classification of test vector $x$ is given by projecting $x$ onto the weight vector $w$ as shown in Eq. (4).

$y=w^{T} x_{\text {test }}$

Given a training set of data vectors $x$, a projection that maximizes the class separation for two classes $C 1$ and $C 2$ is obtained to find $w$. A good separation should be given, when the projections of the class means exposing a long distance along the direction of $w$. These projections are given by Eq. (5), where $m_{i}$ represents the mean vectors of the two classes, and $w$ their projections onto $w$.

$\mu_{1}-\mu_{2}=w^{T}\left(m_{1}-m_{2}\right)$

The classification of a test dataset with FLDA provides each sample, first of all, with the projection of the sample onto $w$ and the class label. The first output corresponds to a gradual decision and the second to a binary decision. The projection is a scalar value that gives a measure of the distance in the projection between the sample and the hyperplane. This distance can be interpreted as a measure for the distinctness of the sample from samples of the other class. The FLDA finds the best separation of two classes by maximizing the quotient of the class mean distance and the class variance. To achieve a good separation, it is desirable to have a large distance between the means.

\section{Open loop tuning for PID controller}

This section describes the procedure to tune a PID controller using the open loop method.
There are a lot of technics to make this aim O'Dwyer (2008), but this article is centered in the one describes in the next point; one of the difference between the technics are the way to study the respond of a system. The result of the open loop tuning, is not achieved directly; it is necessary to process the respond of the system, and use a variety of expression to find the correct constants to define the controller. As the controller is a PID controller, the necessary constants are $K, T_{i}$, and $T_{d}$.

\subsection{First Order Lag Plus Delay system}

The key to find good results of the parameters for the PID controller is to ensure that the system is a First Order Lag Plus Delay (FOLPD). The open loop tuning consist on introduce and step in the system to change its set point. The respond of the system to this new set point, should be anything like the responses in the Fig. 3.

The characteristics of the response $K$ System Gain, $L$ Lag time, and $T$ Response time are obtained (Fig. 3) for a unit step input.

This research use the relation between $L$ and $T$ to decide the best tuning method to the PID controller, according to the parameter of the system that is necessary to perform. The method in this work is only applied to a range from 0 to 1 of the parameter $L / T$.

\subsection{Expressions to define the constants of the PID}

There are a lot of expression to define the constants of a PID controller, base on the empirical open loop tuning respond of a system. As this paper is oriented to an industrial environmental, it is decided to focus only in the load disturbance expressions (Åström and Hägglund, 2009).

The expressions can be grouped depending of the parameter studied by the author to achieved them. Fig. 4 shows the total of expressions used, and the corresponding parameter which are base the authors.

The expressions used to improve the overshoot ratio as a $1 / 4$ per overshoot, mean that every overshoot will be a maximum of $1 / 4$ of the previous overshoot. The expressions in this group are the Ziegler Nichols (Z\& N) and the Chien, Hrones and Reswick (CHR) expressions. It is necessary to remark that there are two different expressions of CHR depending of the theoretical overshoot allowed to the system.

The Kaya and Scheib (K\& S) expressions are three depending of the factor to improve. IAE means that they studied the integral of the absolute error in the system respond. In ISE, the studied parameter was the integral value of the square of the error. ITAE means they studies the integral of the absolute error multiplied by the time.

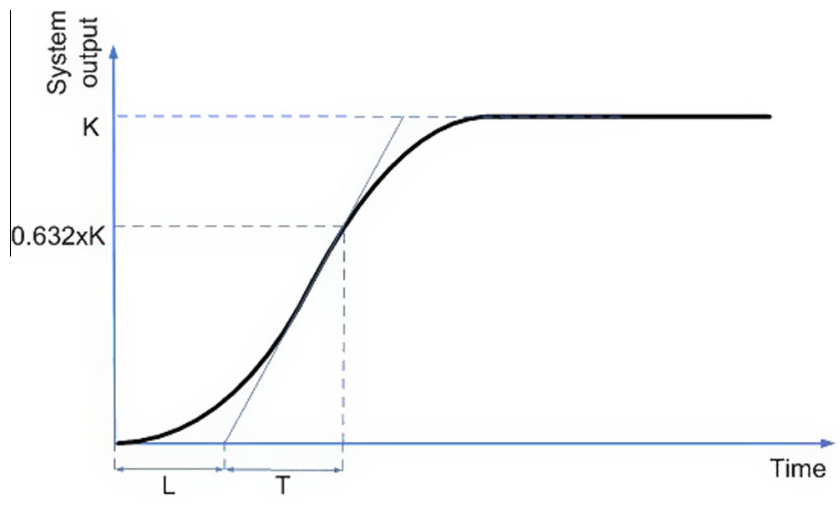

Fig. 3. System Response to step input. 


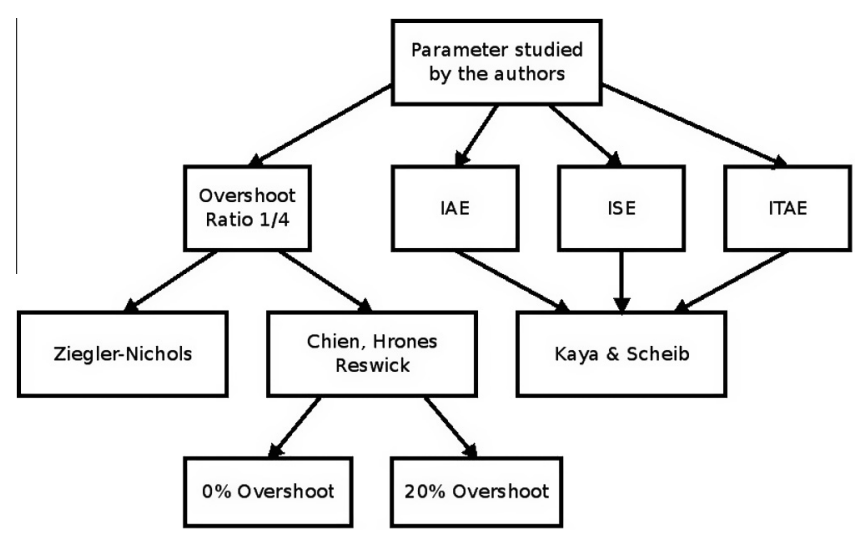

Fig. 4. Expressions used for tuning the PID.

The expressions used in this article are defined in Table 1.

\section{Hybrid system}

The general structure of the control system is shown in Fig. 5, where the input of the system is applied to the controller selected by the hybrid system.

The Hybrid System is composed by 2 main blocks (Fig. 6):

- The "Knowledge of existing rules": This block organize the existing knowledge about the systems in a general flow-chart, which determines which intelligent model must be applied for selecting the best controller.

- The "Intelligent models": This block is composed by 2 intelligent models. The first one considers the controllers "K\& S_IAE, K\& S_ISE, K\& S_ITAE" and the second one considers the controllers "Z\& N, CHR0\%, CHR20\%".

\subsection{Knowledge of existing rules block}

The general flow-chart of this block is shown in Figs. 7 and 8. In this part of the general flow-chart (Fig. 7), the first task to be done is to inquire whether the system is apt for PID open loop tuning methods. Then in both cases it is checked whether the response system is a First Order Lag Plus Delay (FOLPD), if it is not the case, it would not be possible to carry out controller tuning with this method. After having checked if it is a first order with time delay system response, if it is not the case, this design method will not be applied.

If it is a FOLD system, "L/T" (dimensionless) is checked to see if it is bigger than 0.1 (empirical value (Åström and Hägglund, 2009)), if positive, it can be used the expressions contemplated in the first rule rg.1 (Z\& N, CHR0\%, CHR20\%). If it is lower than 0.1 only the expression contemplated in the second rule can be used (K\& S_IAE, K\& S_ISE, K\& S_ITAE).

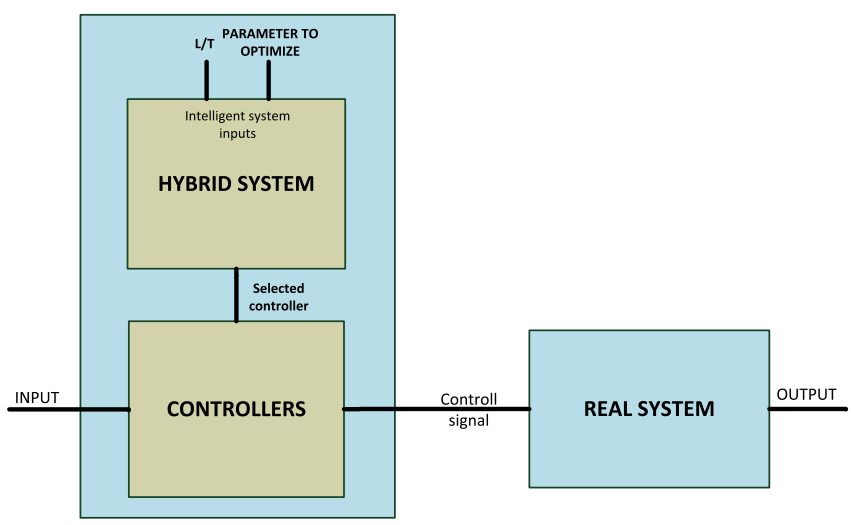

Fig. 5. General structure of the Control System.

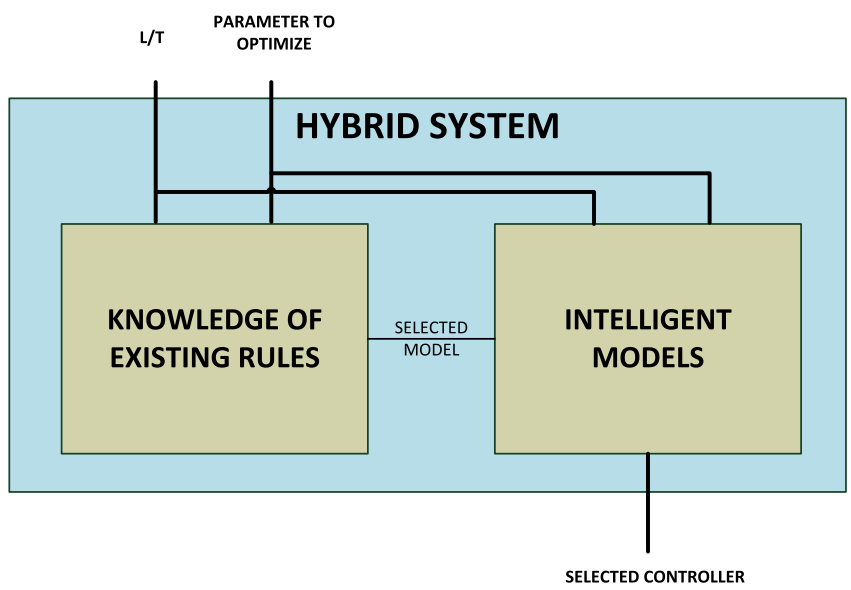

Fig. 6. General struture of the Hybrid System.

The corresponding part of the diagram of Fig. 8 is employed to discover if the system response is a first order lag plus time delay (FOLPD) system. Two steps are necessary followed for it. The first step is to check if the system stabilizes at a constant value with a unit step input, and that there is no oscillation. If so, the next step is to make sure if there is a system of FOLPD type. If both conditions are met, it can be concluded that the system is of this type, otherwise it is not.

\subsection{Intelligent models block}

This block contains the best models classifiers generated by using ANN, SVR-LR and FLDA. During the training process, these models receives 2 inputs "L/T" and "parameter to improve" and one output "best controller" selected by an expert empirically. All algorithms are trained for both rules (rg.1 and rg.2):

Table 1

Expressions used in the study ( $K \& S$ ).

\begin{tabular}{|c|c|c|c|}
\hline Expressions & $K_{p}$ & $T_{i}$ & $T_{d}$ \\
\hline Z\& N Ziegler and Nichols (1993) & $1.2 \frac{T}{K L}$ & $2 L$ & $0.5 L$ \\
\hline CHR0\% $M_{p}$ Chien et al. (1952) & $\frac{0.95}{a}$ & $2.4 L$ & $0.42 L$ \\
\hline CHR20\% $M_{p}$ Chien et al. (1952) & $\frac{1.2}{a}$ & $2 L$ & $0.42 L$ \\
\hline K\&S_IAE Kaya and Scheib (1988) & $\frac{0.98089}{K}\left(\frac{T}{L}\right)^{0.76167}$ & $\frac{T}{0.91032}\left(\frac{T}{L}\right)^{1.05221}$ & $0.59974 T\left(\frac{L}{T}\right)^{0.89819}$ \\
\hline K\&S_ISE Kaya and Scheib (1988) & $\frac{1.11907}{K}\left(\frac{T}{L}\right)^{0.89711}$ & $\frac{T}{0.7987}\left(\frac{T}{L}\right)^{0.9548}$ & $0.54766 T\left(\frac{L}{T}\right)^{0.87798}$ \\
\hline K\&S_ITAE Kaya and Scheib (1988) & $\frac{0.77902}{K}\left(\frac{T}{L}\right)^{1.06401}$ & $\frac{T}{1.14311}\left(\frac{T}{L}\right)^{0.70949}$ & $0.57137 T\left(\frac{L}{T}\right)^{1.03826}$ \\
\hline
\end{tabular}


- Rule 1 (rg.1): When the "Knowledge of existing rules block" determines that rule 1 must be applied, in this case only controllers "K\& S_IAE, K\& S_ISE, K\& S_ITAE" are applicable.

- Rule 2 (rg.2): When the "Knowledge of existing rules block" determines that rule 2 must be applied, in this case only controllers Z\& N, CHRO\%, CHR20\%, are applicable.

For both situations, all algorithms are trained and the best model, in terms of minimum error is selected as the model to use in the "models block".

\subsection{Dataset}

The following datasets were used for developing each model for rg. 1 and rg.2:

- For rg.1: The complete dataset consists of 2408 samples, two third of them are used for training (1605), while the remaining third is used for testing (803).

- For rg.2: The complete dataset consists of 7144 samples, two third of them are used for training (4763), while the remaining third is used for testing (2381).

Both datasets have the following inputs:

- L/T: Ratio from 0 to 0.1 for rg.1 and from 0.1 to 1 for rg.2.

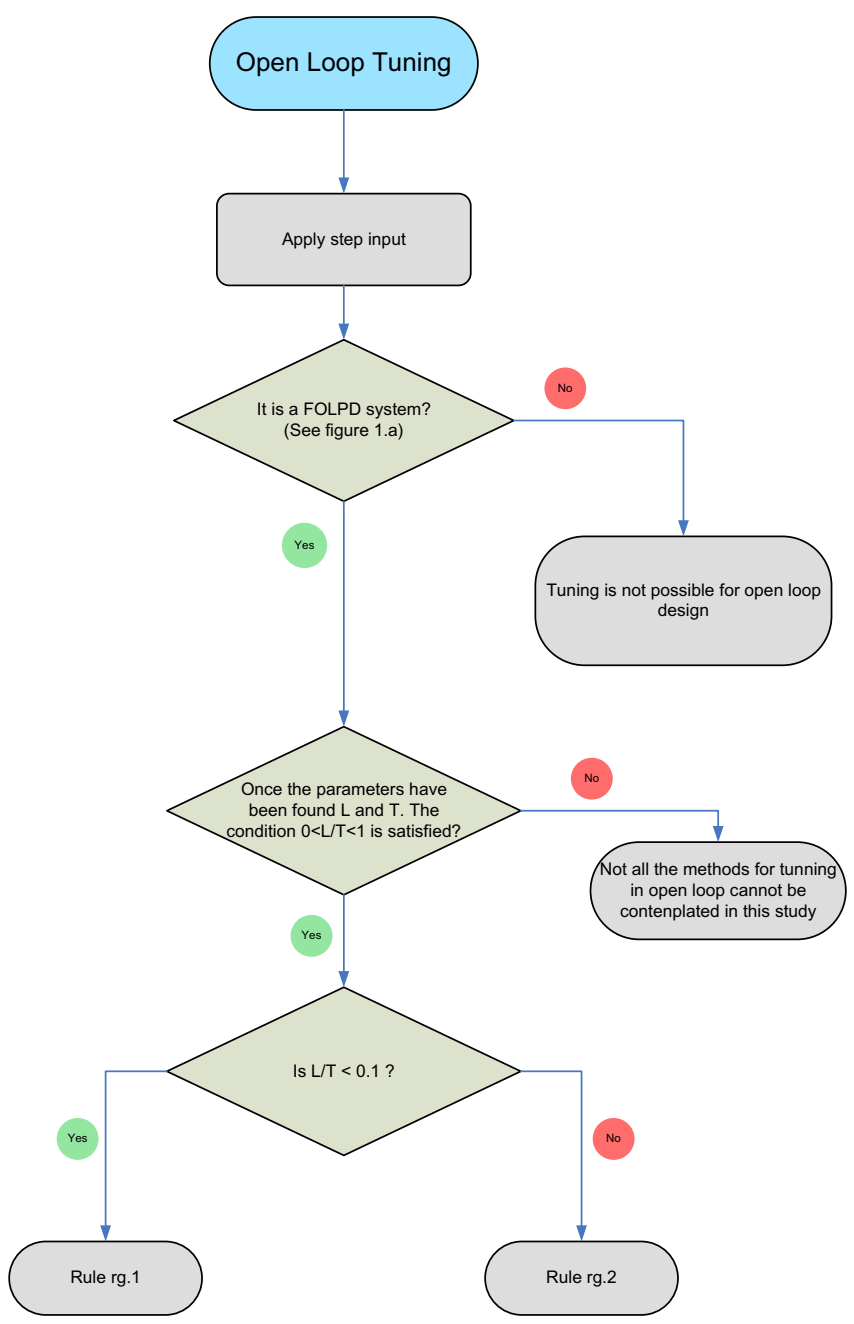

Fig. 7. First Part of General Flow-Chart of Knowledge.
- Parameter to improve: Is de parameter for which the selected controller has to get the best result (Response time $=1$, Overshoot $=2$, Peak time $=3$, Settling time $=4$ ).

And one output:

- Best controller: $Z \& N=1, C H R 0 \%=2, C H R 20 \%=3, K \& S \_I A E=4$, $\mathrm{K} \& \mathrm{~S} \_\mathrm{ISE}=5, \mathrm{~K} \& \mathrm{~S} \_\mathrm{ITAE}=6$. The selection of the best controller was performed by an expert basing on the response of the system comparing all possible controllers.

\subsection{Experiments and results}

For each rule, all algorithms (ANN, LS-SVM and FLDA) were trained using a cross validation of 10 folds.

- ANN: MLP was used. It was tested with 5, 10 and 15 neurons in the hidden layer, log sigmoidal and tangent sigmoidal transfer functions for the hidden layer, and linear for the output layer. To train the MLP, the Levenberg-Marquardt optimization algortihm (Levenberg, 1944) was used to update the weights and bias of the network, as it is often faster than classical error backpropagation algorithm (Matworks, 2013). Finally the best results were obtained using 10 neurons and a sigmoidal transfer function for the hidden layer.

- SVM: LS-SVM (Least Square Support Vector Machine) (DeBrabanter, 2013) Matlab toolbox was used. In this toolbox, the tuning of the parameters is conducted in two steps. First, a state-of-the-art global optimization technique, Coupled Simulated Annealing (CSA) (Xavier de Souza, 2010), determines suitable parameters according to specific criterion. These parameters are then given to a second optimization procedure (simplex or gridsearch) to perform a fine-tuning step.

Following, the results of the three algorithms are shown in Table 2 (confusion matrices for rg.1) and Table 3 (confusion matrices for rg.2).

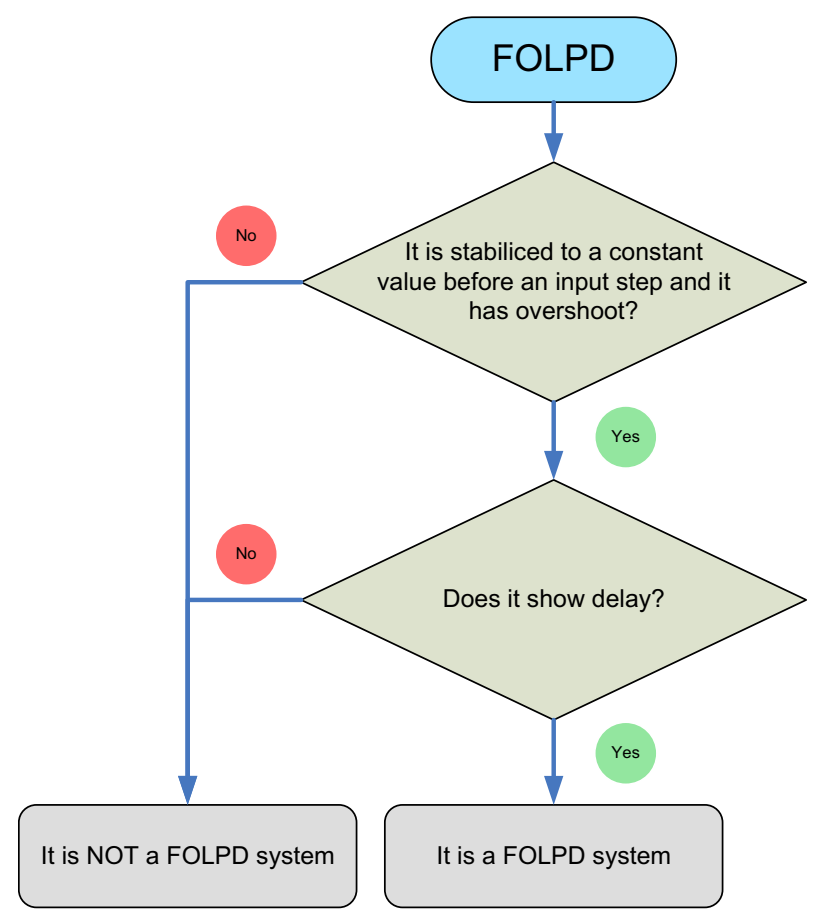

Fig. 8. Second Part of the General Flow-Chart of Knowledge to find out if a system is a FOLD system type. 
Table 2

Results for rule rg.1.

\begin{tabular}{|c|c|c|c|}
\hline \multirow[t]{2}{*}{ Desired method } & \multicolumn{3}{|c|}{ Method chosen by model } \\
\hline & K\& S_IAE & K\& S_ISE & K\& S_ITAE \\
\hline \multicolumn{4}{|l|}{ (a) FLDA } \\
\hline K\& S_IAE & 146 & 58 & 63 \\
\hline K\& S_ISE & 65 & 150 & 53 \\
\hline K\& S_ITAE & 63 & 49 & 156 \\
\hline SE & 0.547 & 0.560 & 0.582 \\
\hline ACC & 0.690 & 0.720 & 0.716 \\
\hline \multicolumn{4}{|l|}{ (b) $L S-S V M$} \\
\hline K\& S_IAE & 214 & 30 & 23 \\
\hline K\& S_ISE & 34 & 207 & 27 \\
\hline K\& S_ITAE & 32 & 24 & 212 \\
\hline SE & 0.801 & 0.772 & 0,791 \\
\hline ACC & 0.852 & 0.857 & 0.868 \\
\hline \multicolumn{4}{|l|}{ (c) $M L P$} \\
\hline K\& S_IAE & 253 & 6 & 8 \\
\hline K\& S_ISE & 6 & 260 & 2 \\
\hline K\& S_ITAE & 2 & 5 & 261 \\
\hline SE & 0.948 & 0.970 & 0.974 \\
\hline ACC & 0.973 & 0.976 & 0.979 \\
\hline
\end{tabular}

Table 3

Results for rule rg.2.

\begin{tabular}{llll}
\hline \multirow{2}{*}{ Desired method } & \multicolumn{2}{l}{ Method chosen by model } \\
\cline { 2 - 4 } & Z\& N & CHR\%0 & CHR\%20 \\
\hline (a) FLDA & & & \\
Z\& N & 461 & 167 & 165 \\
CHR\%0 & 163 & 492 & 139 \\
CHR\%20 & 161 & 194 & 439 \\
SE & 0.581 & 0.620 & 0.553 \\
ACC & 0.724 & 0.722 & 0.723 \\
(b) LS-SVM & & & \\
Z\& N & 642 & 79 & 72 \\
CHR\%0 & 56 & 671 & 67 \\
CHR\%20 & 93 & 54 & 647 \\
SE & 0.810 & 0.845 & 0.815 \\
ACC & 0.874 & 0.892 & 0.880 \\
(c) MLP & & & \\
Z\& N & 756 & 17 & 20 \\
CHR\%0 & 17 & 765 & 12 \\
CHR\%20 & 19 & 34 & 741 \\
SE & 0.953 & 0.963 & 0.933 \\
ACC & 0.969 & 0.966 & 0.964 \\
\hline
\end{tabular}

These matrices also show the Sensitivity (SE) and the Accuracy (ACC).

$$
\begin{aligned}
& S E=\frac{T P}{(T P+F N)} \\
& A C C=\frac{T P+T N}{(T P+T N+F P+F N)}
\end{aligned}
$$

Where TP is the number of True Positive, TN is the number of True Negative, FN is the number of False Negative and FP is the number of False Positive.

Results show that the best classifier in both situations (rg.1 and rg.2) is the MLP, obtaining good results in terms of ACC and SE. The worst classifier is FLDA with a great difference with respect to the ohter two classifiers.

\section{Empirical verification on steel rolling process}

Through the steel foundry cast profile shapes are obtained ingot (billet) as well as through continuous casting machines. Usually this product is stored for further processing according to customer demand.
The transformation of this product consists essentially of the material lamination into profiles directly useable, commercial shapes that have certain properties. Lamination is a volumetric deformation process which reduces the initial section of the material worked by the compressive forces exerted on a piece of metal in between two rollers.

The rollers rotate in opposite directions for the flow of material between them, exerting compressive forces and shearing caused by the friction produced between the rolls and metal. In Fig. 9 shows a simplified diagram of what happens in each of the roll stands of the disposal of the rolling mill.

Following the implementation of the process it has to be performed the speed control, which is a critical parameter for a correct rolling.

The speeds to be programmed into the cylinders of different boxes have to be very precise and adjusted to prevent disturbances in order to achieve an optimum material output quality. For explanation and correct comprehension of the so adopted solution it is necessary to take into account that the relationship between the area of the incoming section $\left(S_{i}\right)$ and the area of the outgoing section $\left(S_{o}\right)$, it is proportional to the occurring elongation of the material as it passes in between the two rollers of the box. Thus the section is reduced at the expense of making longer the piece.

Since the amount of material does not vary, it is defined the reduction factor $R$ as the ratio between the area of material entering and leaving the rollers. The same factor can be obtained by relating the linear speeds of input and output of material. In Eq. (8) both discussed relationships are stated.

$R=\frac{S_{i}}{S_{o}}=\frac{V_{o}}{V_{i}}$

\subsection{Control case study}

On this issue it is proposed that the reduction factor $R$ is the data that allows for the speed setting point of the different boxes of a rolling mill. Thus, if the reduction factors of the different boxes $R$ train have been carefully selected simply by varying the factors if necessary, the speed setting points will be automatically updated at all points.

In the rolling mill to be monitored there are seven gearboxes.

The feeding of the process is performed with material whose section is 22500 square millimeters, and it is intended a production speed of the final product of 2.30 meters per second.

Based on the desired speed, the characteristics of the starting material and the reduction factors of each of the boxes of the rolling train, taking into account the expression (8), we obtain the linear speeds of input and output of material to rollers.

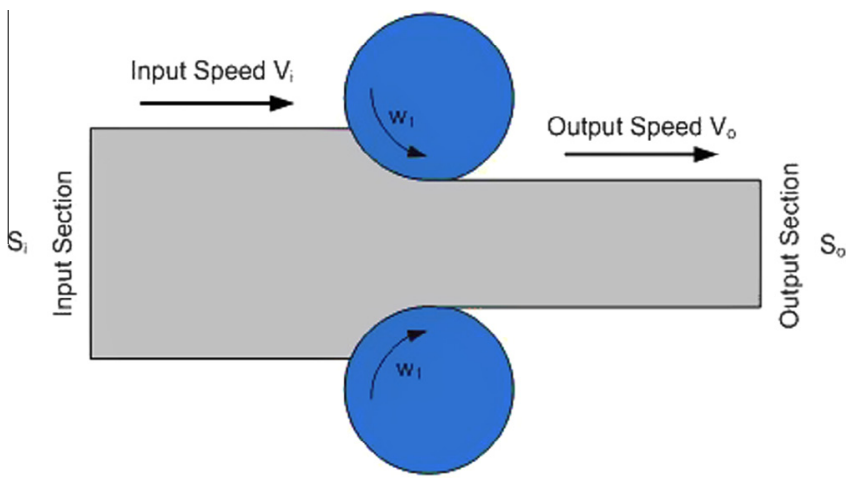

Fig. 9. Outline of the laminating rollers. 
Table 4

Summary of reduction factor, sections and mill speeds.

\begin{tabular}{llllll}
\hline & R Factor & Input $\mathrm{mm}^{2}$ & Output $\mathrm{mm}^{2}$ & Input $\mathrm{m} / \mathrm{s}$ & Output $\mathrm{m} / \mathrm{s}$ \\
\hline Box 1 & 1.456 & 22500.0 & 15453.0 & 0.40 & 0.58 \\
Box 2 & 1.425 & 15453.0 & 10844.0 & 0.58 & 0.83 \\
Box 3 & 1.375 & 10844.0 & 7886.9 & 0.83 & 1.14 \\
Box 4 & 1.311 & 7886.9 & 6015.9 & 1.14 & 1.49 \\
Box 5 & 1.258 & 6015.9 & 4782.1 & 1.49 & 1.87 \\
Box 6 & 1.123 & 4782.1 & 4257.3 & 1.87 & 2.10 \\
Box 7 & 1.094 & 4257.3 & 3892.1 & 2.10 & 2.30 \\
\hline
\end{tabular}
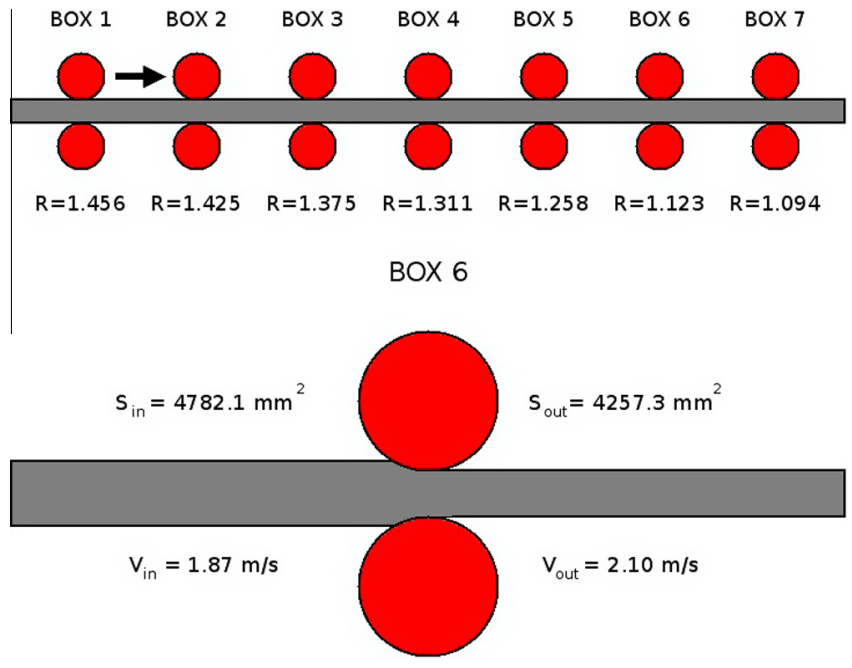

Fig. 10. Diagram of rolling mill with boxes and reduction factors.

Table 4 lists all the parameters of the rolling mill.

The speed control is carried out upstream in the opposite direction to the flowing material.

The starting point is the output speed indicated by the designer and mechanical installation, based on the reductions of the boxes, where different linear velocities of the material at all points are obtained. These velocities correspond to the section reductions that occur as the material progresses in line for the various boxes.

In Fig. 10 different boxes are shown as an example, where it is indicated for the box No. 6, sections and input and output speeds of the material. Also in all of them, apart from the identification the different real reduction factors obtained after the different tests applied to the process are shown.

It is important to highlight that the reduction factor need not be stable, in fact, what is occurring is a "flattening" of the material and, for example, its value depends on the temperature.

Thus for example if the temperature of the leaving bar from the reheating furnace is slightly higher than usual, that bar tends to deform in a different way and, consequently, the output speed will be different. This means that the boxes must be perfectly synchronized regarding speeds, otherwise there are two possible cases:

1. The following box is faster: If this is the case there will be a sliding of the roller that performs the laminate, with the consequent wear and increase in temperature among the others.

2. The following box is slower: If this is happened the material will be bent and deformed in a different direction to the displacement of the rolling mill and can form dangerous accumulations.

\subsection{Results - findings}

Depending on the diameter of the gearbox and the target linear speed, we can obtain the speed at which the actuator must rotate, and that means the velocity to be programmed in each of them.

This method ensures that varying one factor reduction rates are automatically updated throughout, and thus will not have the problems that had been occurring in these processes, i.e. the need to retrain all speeds separately.

The adjustment of the servos are to be carried out with the loaded plant in operation, otherwise the installation would not perform well.

The adjustment of PID controllers of the servos are to be performed in an open loop, through a procedure that favors disturbance rejection. It is to be loaded and set to the velocity for which the train has been designed.

In addition to applying the model of this developed knowledge, they have been taken into account the following principles in order to standardize the response and for the results to be optimal:

1. Only terms whose criterion is related to disturbance rejection have been taken into account, in spite that apparently would bring a worse answer, but nevertheless, it provides much better results against possible disturbances or load variations. This is the reason why the terms used are listed in Table 1.

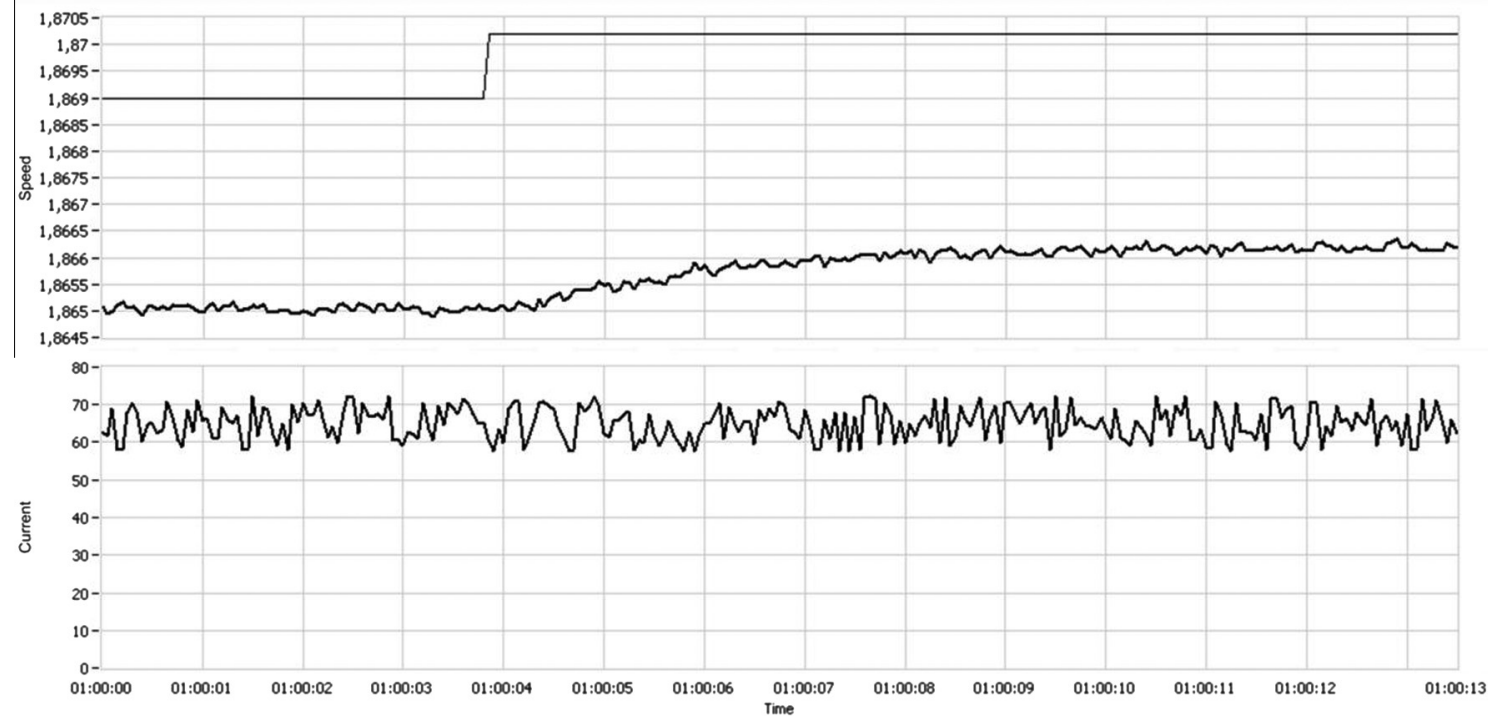

Fig. 11. Example of response measurement for adjusting servomotor. 
2. In all cases the same expressions are applied across the rolling mill even though better specifications could be achieved through different expressions for every box in the mill.

3. Although the model is general, it has expressions to be followed for setting point and disturbance rejection purposes. It was designed ad hoc for the last case as it is crucial in this system we intend to adjust.

4. Although the rolling mill is dimensioned from a mechanical point of view for an output speed of material 2.3 meters per second, synchronizing tests have been carried out starting at 2 meters per second.

A probe is put under load (with material between the rollers) of a known magnitude which will produce the parameters to be introduced into the expressions.

Adjustments are made for material output speeds of 2.0, 2.1, 2.2 and $2.3 \mathrm{~m} / \mathrm{s}$.

For every case the starting point is a steady speed slightly lower than the laminate one and subsequently turned onto the corresponding rolling speed.
An example in Fig. 11 BOX 6 corresponding to the rolling train is shown.

After analyzing the graph of Fig. 11 it is obtained a lag $L$ of $1.2 \mathrm{~s}$ and a rise time $T$ of $3.4 \mathrm{~s}$. There is thus a parameter $L / T$ of 0.353 , which is applied as input to model knowledge.

The expressions for the disturbance rejection criterion that indicates this knowledge model are those of Chien, Hrones \& Reswick.

If you were to perform a test in which a rod is inserted in the rolling mill BOX 6 the answer would be according to Fig. 12.

In the Fig. 12 it is observed that the linear velocity corresponding to the rotation before entering the bar is greater than the required $1.87 \mathrm{~m} / \mathrm{s}$. To perform the adjustment test it is given as setting guidance of $1.869 \mathrm{~m} / \mathrm{s}$ and when approximately half of the bar has been rolled then is set regularly to $1.8702 \mathrm{~m} / \mathrm{s}$.

As for the motor current can be observed that consumption is very low when unloaded, while in load is very high, and although slightly increased the speed it is not perceived any change in the current consumption.

It should be noted that with respect to the reference set, the speed decreases instantaneously when a bar comes in and drags,

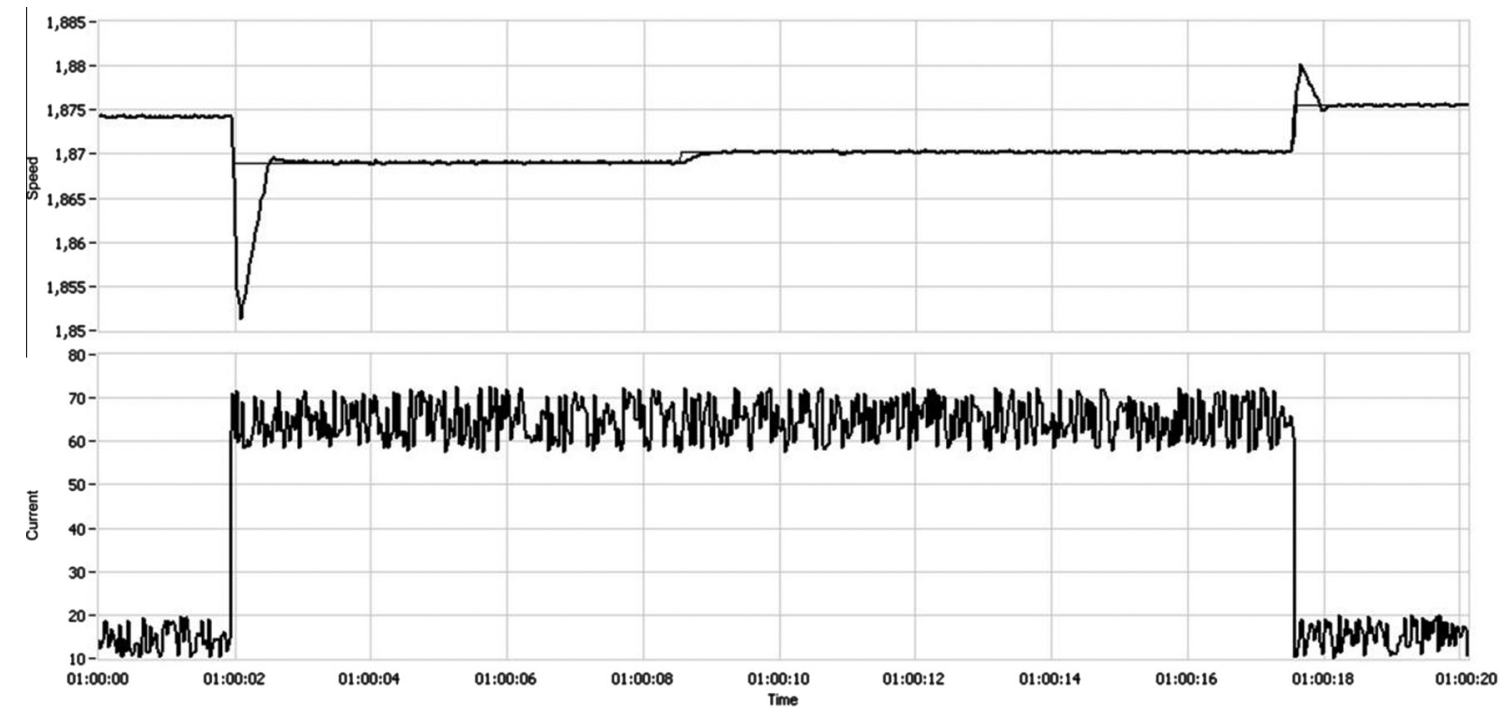

Fig. 12. Speed and current in BOX 6 when a bar enters tuned by CHR.

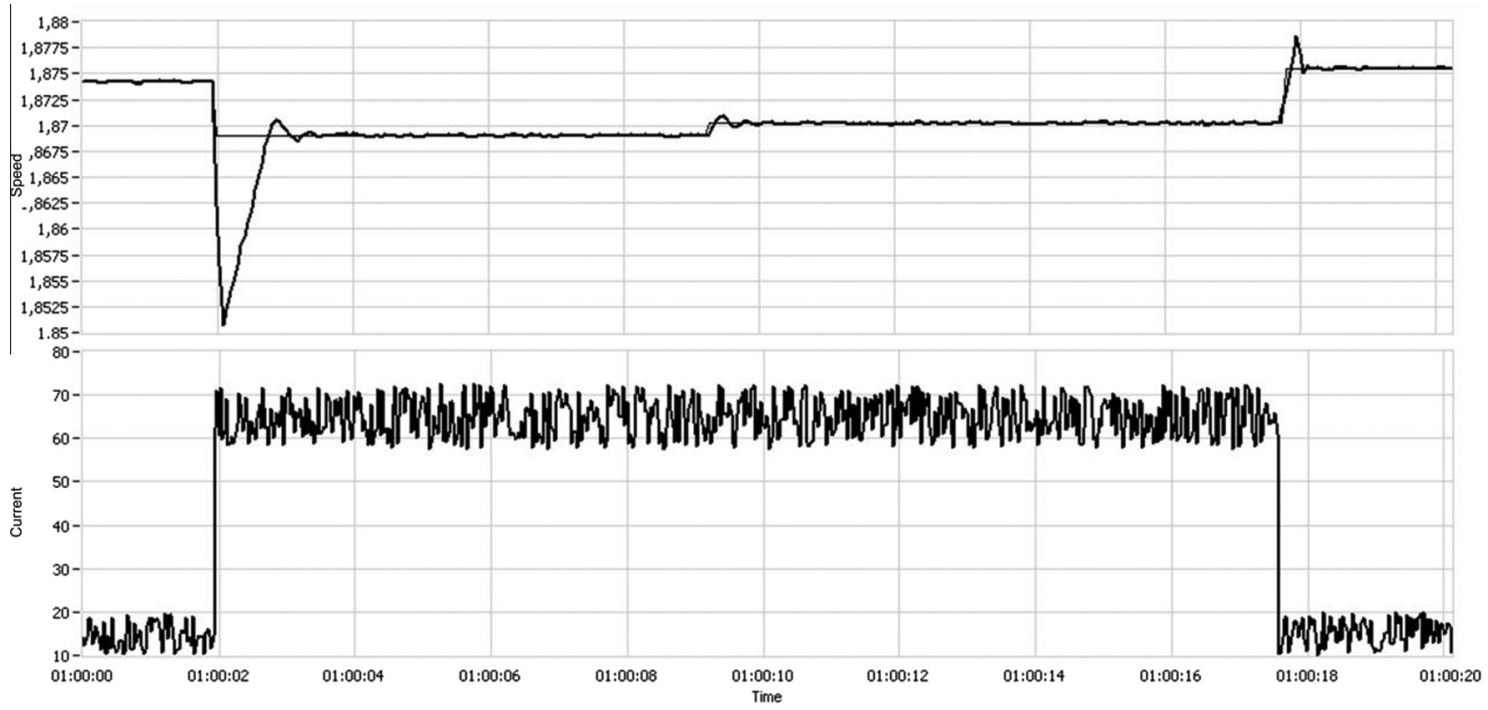

Fig. 13. Speed and current in BOX 6 when it enters a bar tuned by Z\& N. 
and when a bar is released there is also a considerable acceleration, but after the transient setting point it follows correctly and satisfactorily.

If the parameters in the controller were typical of Ziegler-Nichols, the answer would be according to Fig. 13, which as it can be seen, it is not as satisfactory as the selected model.

\section{Conclusions}

The proposed method allows to obtain the optimal performance of the steel rolling process by selecting the best parameters of the PID controller. The system, based on knowledge rules and ANN, selects the most adequate expression for calculating the PID parameters in an automatic way, without the knowledge of expert operators. It makes easier to start up the process on which the developed system is applied.

The model was tested on real steeling roll process with a unknown transfer function. The results obtained are presented in the Results - Findings section to fulfill the initial objectives by verifying the correct behavior of the developed system. Therefore, the Hybrid Model allows to improve the transient and permanent regimes of the process response.

From a general point of view of the Hybrid Model, three useful contributions were made. Firstly, greater clarity was achieved for various stages in the adjustment of a PID design. Secondly, certain contradictions were shown up between different methods, these being solved by the use of the model. Thirdly, a method for the automatic tuning of PID to control systems in open loop has been described, allowing to get a finer grain model based on data-mining and techniques.

\section{Acknowledgment}

This research is partially supported by Predoctoral grant of "Xunta de Galicia Goverment".

\section{References}

Åström, K. J., \& Hägglund, T. (2009). Advanced pid control. Pearson Education.

Alvarez-Huerta, A., González-Miguélez, R., \& García-Metola, D. (2011). Drywell temperature prediction of a nuclear power plant by means of artificial neural networks. DYNA Ingeniera e Industria, 86, 467-473.

Astrom, K. J., \& Wittenmark, B. (1994). Adaptive control (2nd ed.). Boston, MA, USA: Addison-Wesley Longman Publishing Co., Inc..

Bahill, A. (1983). A simple adaptive smith-predictor for controlling time-delay systems: A tutorial. IEEE Control Systems Magazine, 3, 16-22.

Belhumeur, P. N., Hespanha, J. P., \& Kriegman, D. J. (1997). Eigenfaces vs. fisherfaces: Recognition using class specific linear projection. IEEE Transactions on Pattern Analysis and Machine Intelligence, 19, 711-720.

Bishop, C. M. (2006). Pattern recognition and machine learning. Springer.

Camacho, E., \& Bordons, C. (2004). Model predictive control. Advanced textbooks in control and signal processing. Springer.

Chang, N.-B., Pongsanone, N. P., \& Ernest, A. (2011). Comparisons between a rulebased expert system and optimization models for sensor deployment in a small drinking water network. Expert Systems with Applications, 38, 10685-10695.

Chaoui, H., Sicard, P., Lakhsasi, A., \& Schwartz, H., (2004). Neural network based model reference adaptive control structure for a flexible joint with hard nonlinearities. In Industrial Electronics, 2004 IEEE on International Symposium (Vol. 1, pp. 271-276).

Chen, Y., Zhang, J., Chai, T., Fu, J., \& Ding, J., (2010) Hybrid intelligent control of heating furnaces for steel rolling process. In 2010 8th World Congress on, Intelligent Control and Automation (WCICA) (pp. 2674-2679).
Chen, C.-H., Lin, C.-M., \& Chen, T.-Y. (2008). Intelligent adaptive control for mimo uncertain nonlinear systems. Expert Systems with Application, 35, 865-877.

Chien, K. L., Hrones, J. A., \& Reswick, J. B. (1952). On the automatic control of generalized passive systems. Transations of the ASME, 175-185.

Cimino, M. G. C. A., Lazzerini, B., Marcelloni, F., \& Ciaramella, A. (2012). An adaptive rule-based approach for managing situation-awareness. Expert Systems with Application, 39, 10796-10811.

Cristianini, N., \& Scholkopf, B. (2002). Support vector machines and kernel methods. The new generation of learning machines. AI Magazine, 23, 31-41.

DeBrabanter, et al., (2013).LS-SVMlab Toolbox User's Guide version 1.7., Technical Report, Matworks

Etik, N., Allahverdi, N., Sert, I. U., \& Saritas, I. (2009). Fuzzy expert system design for operating room air-condition control systems. Expert Systems with Application, 36, 9753-9758.

Ferreiro-Garcia, R. (2012). Improving heat exchanger supervision using neural networks and rule based techniques. Expert Systems with Applications, 39, 3012-3021.

Garliauskas, A. (2004). Conceptions and modeling for transmitted information evaluation by ann. Informatica, Lithuanian Academy of Science, 15, 475-488.

Haddad, W., \& Chellaboina, V. (2011). Nonlinear dynamical systems and control: A lyapunov-based approach. Princeton University Press.

Hayes-Roth, F. (1985). Rule-based systems. Communication of the ACM, 28, 921-932.

Hayes-Roth, F., Waterman, D., \& Lenat, D. (1983). Building expert systems. AddisonWesley Pub. Co..

Hornik, K., Stinchcombre, M., \& White, H. (1989). Multilayer feedforward networks are universal approximators. Neural Networks, 2, 359-366.

Kaya, A., \& Scheib, T. (1988). Tuning of pid controllers of different structures. Control Engineering, 62-65.

Ko, H.-H., Kim, J.-S., Kim, J., Baek, J.-G., \& Kim, S.-S. (2011). Intelligent adaptive process control using dynamic deadband for semiconductor manufacturing. Expert Systems with Application, 38, 6759-6767.

Levenberg, K. (1944). A method for the solution of certain non-linear problems in least squares. Quarterly of Applied Mathematics, 5, 164-168.

Liu, H., Zabinsky, Z., \& Kohn, W. (2010). Rule-based control system design for smart grids. In Power and energy society general meeting (pp. 1-5). IEEE.

Maheral, P., Ide, K., Gomi, T., Pussegoda, N., \& Too, J., (1995). Artificial intelligence techniques in the hot rolling of steel. In Canadian conference on electrical and computer engineering (Vol. 1, pp. 507-510).

Marlin, T. (2000). Process control: Designing processes and control systems for dynamic performance. McGraw-Hill chemical engineering series. McGraw-Hill.

Matworks, (2013). Levenberg-Marquardt backpropagation, Technical Report, Matworks Documentation Center.

O’Dwyer, A., (2006). Pi and pid controller tuning rules: An overview and personal perspective. In Proceedings of the IET Irish signals and systems conference (pp. 161-166).

Olugu, E. U., \& Wong, K. Y. (2012). An expert fuzzy rule-based system for closedloop supply chain performance assessment in the automotive industry. Expert Systems with Applications, 39, 375-384.

Rud, O. C., \& Mining, Data (2000). Cookbook: Modeling data for marketing. Risk and customer relationship management. John Wiley \& Sons, Inc.

Rugh, W. (1991). Analytical framework for gain scheduling. IEEE Control Systems, 11, 79-84

Sastry, S., \& Bodson, M. (2011). Adaptive control: Stability, convergence and robustness. Dover books on electrical engineering series. Dover Publications.

Sbarbaro-Hofer, D., Neumerkel, D., \& Hunt, K. (1993). Neural control of a steel rolling mill. IEEE Control Systems, 13, 69-75.

Srivastava, L., Singh, S., \& Sharma, J. (1999). Knowledge-based neural network for voltage contingency selection and ranking. IEE Proceedings-Generation, Transmission and Distribution, 146, 649-656.

Stanikunas, R., \& Vaitkevicius, H. (2000). Neural network for color constancy. Informatica, Lithuanian Academy of Science, 11, 219-232.

Swets, D. L., \& Weng, J. J. (1996). Using discriminant eigenfeatures for image retrieval. IEEE Transactions on Pattern Analysis and Machine Intelligence, 18 831-836.

Wan, Z., Wang, X., \& Wu, J., (2008). Model adaptive learning for steel rolling mill control. In IEEE international symposium on knowledge acquisition and modeling workshop, 2008. KAM Workshop (2008) (pp. 903-906).

Xavier de Souza, S., et al., (2010). Coupled simulated annealing, In IEEE Transactions on Systems, Man and Cybernetics - Part B (Vol. 40-2, pp. 320-335).

Ye, J., \& Xiong, T., (2008). Svm versus least squares svm. In: 11th International conference on artificial intelligence and statistics (AISTATS), (pp. 640-647).

Ziegler, J. G., \& Nichols, N. B. (1993). Optimum settings for automatic controllers. Journal of Dynamic Systems, Measurement, and Control, 115, 220-222. 